\title{
Prospecção tecnológica do uso de surfactantes como aditivo no biodiesel
}

\author{
Technological prospection of the use of surfactants as an additive in biodiesel \\ Prospección tecnológica del uso de tensioactivos como aditivo del biodiesel
}

Recebido: 23/08/2021 | Revisado: 29/08/2021 | Aceito: 01/09/2021 | Publicado: 04/09/2021

Natália de Oliveira Mascarenhas
ORCID: https://orcid.org/0000-0002-6970-0078
Universidade Federal da Bahia, Brasil
E-mail: mascarenhas.ndo@ gmail.com
Maurício de Almeida Pereira
ORCID: https://orcid.org/0000-0001-7035-1824
E-mail: mauriciopereira.ufba@ gmail.com
George Simonelli
Instituto Federal de Educaco, Ciência Tecnogia
ORCID: https://orcid.org/0000-0002-8031-1401
Universidade Federal da Bahia, Brasil
E-mail: gsimonelli @ufba.br
Luiz Carlos Lobato dos Santos
ORCID: https://orcid.org/0000-0003-3824-7802
Universidade Federal da Bahia, Brasil
E-mail: lclsantos@ufba.br

\section{Resumo}

O biodiesel tem se mostrado altamente promissor na substituição parcial ou total do óleo diesel derivado do petróleo, sendo a reação de transesterificação pela via metílica a mais utilizada. No entanto, a diferença de polaridade dos reagentes causa dificuldades na homogeneização do sistema reacional, o que pode levar a perdas de rendimento. Esse problema pode ser minimizado com o uso de surfactantes. O presente trabalho possui como objetivo prospectar tecnologias voltadas para a utilização de surfactantes como aditivos em biodiesel, a partir da busca na base de dados do depósito de patentes do European Patent Office (EPO) e do Instituto Nacional de Propriedade Industrial (INPI). A pesquisa foi realizada em julho de 2021, sendo encontradas e analisadas as patentes depositadas no período de 2000 a 2020, considerando um estudo documental. Foi possível observar que o banco de dados da EPO possui mais patentes relacionadas com o tema do que o do INPI. Além disso, a maioria dos documentos encontrados na base de dados da EPO possuem o código de classificação C10L1. A pesquisa na base de dados da EPO demonstrou que há um número significativo de patentes nessa área, distribuídas em 7 diferentes países, demonstrando ser uma área muito promissora para investimentos e desenvolvimento de tecnologias.

Palavras-chave: Biodiesel; Surfactante; Prospecção tecnológica.

\begin{abstract}
Biodiesel has been highly promising in the partial or total replacement of petroleum-derived diesel oil and the methyl transesterification reaction is the most used route. However, the difference in polarity of the reactants causes difficulties in the homogenization of the reaction system, which can lead to yield losses. This problem can be minimized with the use of surfactants. The present work aims to prospect technologies for the use of surfactants as additives in biodiesel, based on a search in the patent filing database of the European Patent Institute (EPO) and the National Institute of Industrial Property (INPI). The research was carried out in July 2021, and patents filed between 2000 and 2020 were found and analyzed, considering a documentary study. It was possible to observe that the EPO's database has more patents related to the subject than the INPI's. In addition, most documents found in the EPO database has the classification code C10L1. The search in the EPO database showed that there is a significant number of patents in this area, distributed in 7 different countries, proving to be a very promising area for investments and technology development.
\end{abstract}

Keywords: Biodiesel; Surfactant; Technological prospect.

\section{Resumen}

El biodiésel ha demostrado ser muy prometedor en la sustitución parcial o total del gasóleo derivado del petróleo. La reacción de transesterificación por la ruta del metilo es la más utilizada. Sin embargo, la diferencia de polaridad de los reactivos provoca dificultades en la homogeneización del sistema de reacción, lo que puede conducir a pérdidas de rendimiento. Sin embargo, este problema se puede minimizar con el uso de tensioactivos. El presente trabajo tiene como objetivo la prospección de tecnologías para el uso de tensioactivos como aditivos en biodiesel, a partir de una búsqueda en la base de datos de presentación de patentes de la Oficina Europea de Patentes (EPO) y el Instituto Nacional de Propiedad Industrial (INPI). La investigación se realizó en julio de 2021, y se encontraron y analizaron 
las patentes presentadas desde 2000 hasta 2020, considerando un estudio documental. Se pudo observar que la base de datos de la EPO tiene más patentes relacionadas con el tema que las del INPI. La encuesta informó que la mayoría de los documentos que se encuentran en la base de datos de la EPO tienen el código de clasificación C10L1. Es posible analizar que la investigación en la base de datos de la EPO mostró que existe un número significativo de patentes en esta área, distribuidas en 7 países diferentes, resultando ser un área muy prometedora para la inversión y el desarrollo tecnológico.

Palabras clave: Biodiesel; Surfactante; Prospección tecnológica.

\section{Introdução}

Atualmente, há o aumento da preocupação com o constante crescimento da utilização de fontes não renováveis de energia, já que há a diminuição das reservas de petróleo, além disso, há a necessidade da diminuição da emissão de gases poluentes. Devido a esses fatores, o estudo e utilização de combustíveis alternativos e renováveis, para motores a diesel, está se tornando cada vez mais intenso (Jagtap, Pawar \& Lahane, 2020; Mirhashemi \& Sadrnia, 2020; Ramkumar \& Kirubakaran, 2017).

Dentre os biocombustíveis, o biodiesel tem se destacado, já que pode ser produzido a partir de matérias primas renováveis, como óleos vegetais e gorduras animais (Kalavathy \& Baskar, 2019; Mahlia et al., 2020). Além disso, é biodegradável e emite menos gases de efeito estufa, como o óxido de nitrogênio, dióxido de enxofre e dióxido de carbono, quando comparado com os combustíveis fósseis, tornando-o uma excelente opção para a substituição total ou parcial do diesel a base de petróleo (Booma et al., 2020; Manigandan et al., 2020), já que pode ser utilizado em motores de ignição por compressão.

O biodiesel é uma mistura de monoésteres alquílicos de ácidos graxos de cadeia longa, produzido a partir de óleos ou gorduras, excesso de álcool de cadeia curta, como por exemplo o metanol ou etanol, e catalisador. Os triglicerídeos presentes no óleo ou gordura, podem ser transformados em biodiesel a partir de quatro métodos principais, a pirólise, microemulsão, mistura entre óleos, e a partir da reação de transesterificação, que é o método mais utilizado (Fonseca et al., 2019; Narowska et al., 2019). A reação de transesterificação é quando ocorre a transformação de triglicerídeos em ésteres; estequiometricamente, são necessários três mols de álcool para um mol de triglicerídeo, mas na prática é utilizado o álcool em excesso, para poder aumentar a produção de éster, já que é uma reação reversível, além de ajudar na separação do glicerol (Fonseca et al., 2019; Guimarães et al., 2018).

Devido a diferença de polaridade entre o óleo/gordura e o álcool, durante a produção do biodiesel, há o aparecimento de problemas de homogeneização da mistura, reduzindo a transferência de massa do sistema, e por consequência a diminuição do rendimento da reação (Rodriguez \& Beckman, 2020). Para poder diminuir a dificuldade de contato entre as substâncias polares e apolares, para a produção do biodiesel, têm se investido em diversas tecnologias, como a utilização de microondas, ultrassom, cavitação e surfactantes, melhorando a miscibilidade entre os reagentes, aumentando a taxa de conversão e a eficiência do catalisador, e diminuindo a temperatura da reação (Rodriguez \& Beckman, 2020; Valverdea, Osmierib \& Recasens, 2019; Wong et al., 2019).

Os surfactantes são substâncias orgânicas que podem ser naturais ou sintéticas, e que possuem como principal característica o comportamento anfifílico, já que apresentam uma parte hidrofílica (polar) e uma parte hidrofóbica (apolar) em sua estrutura, sendo solúveis em estruturas semelhantes. Por sua extremidade polar possuir afinidades com substâncias polares e a extremidade apolar possuir afinidades com substâncias apolares, acaba ocasionando o surgimento de micelas que originam emulsões (Leng et al., 2018). A formação da emulsão acarreta na aceleração da reação de transesterificação, aumentando o teor de éster, diminuindo o tempo de reação, a razão molar ácool:óleo e o custo de produção (Leng et al., 2019).

A literatura relata muitos estudos sobre a utilização de surfactantes na produção do biodiesel. Em 2015, Chang e colaboradores, produziram biodiesel utilizando líquidos iônicos dicatiônicos combinados com tensoativos ácidos de Brönsted 
(BASDILs), sendo comprovado que o maior teor de éster foi o da amostra [C12Sb] [Tos]. Foram utilizados $2 \%$ em mol de surfactante, em razão molar etanol:óleo de 1,5:1, a uma temperatura de $60^{\circ} \mathrm{C}$ e 4,5 horas de reação, obtendo uma conversão de $95,7 \%$.

Salam e colaboradores (2016) utilizaram o surfactante dodecil sulfato de sódio (SDS) mais H2SO4 e o dodecil sulfato de zircônio (ZDS) na reação de transesterificação in situ da alga Nannochloropsis occulata para produção de éster metílico de ácido graxo (Fatty Acid Methyl Ester - FAME). O maior rendimento de éster foi encontrado utilizando o surfactante SDS em meio ácido (H2SO4). O teor de éster aumentou de 87\% (sem surfactante) para 98,3\%, utilizando 9\% em massa do surfactante SDS em meio ácido, em 24 horas de reação a $60{ }^{\circ} \mathrm{C}$.

Em um outro estudo, foi utilizado como surfactante o líquido iônico prótico (PIL) 2-hidroxietilamônio hexanoato [2HEA] [Hx] na reação de transesterificação do óleo de soja, com metanol e uma mistura de metanol e etanol. A utilização de $2 \%$ em peso de PIL como surfactante alcançou uma conversão de $92,72 \%$, em 30 min de reação, a $60{ }^{\circ} \mathrm{C}$, a uma razão molar álcool:óleo de 6:1 e com 1,5\% em peso de KOH como o catalisador (Gomez et al., 2019). Trindade e colaboradores (2019) utilizaram o hexanoato de dietilenotriamônio ([DETA] [Hx]) na reação de transesterificação do óleo de soja utilizando metanol. As condições de reação foram a razão molar álcool: óleo de 9:1; 1,5\% em peso de $\mathrm{KOH}$ como o catalisador, e 3\% em peso de surfactante, em 60 min de reação a $60{ }^{\circ} \mathrm{C}$. O maior rendimento de massa foi de $96,21 \%$.

Simonelli e colaboradores (2019) estudaram e compararam dois tipos de surfactantes comerciais, DBB 7104 e DBB 7107, produzidos pela empresa Bolland, para a produção de biodiesel a partir do óleo de soja e metanol. O surfactante DBB 7107, na quantidade de $0,7 \%$ em peso, foi o que mais contribuiu para o aumento do teor total de ésteres metílicos de ácidos graxos no biodiesel, de 97,72\%, em 1\% de peso de $\mathrm{KOH}$ como catalisador, com razão molar de 6:1, em 30 min de reação a 30 ${ }^{\circ} \mathrm{C}$. Ainda em 2019, Leng e colaboradores compararam a utilização de quatro surfactantes comerciais AOT [bis (2-74 etilhexil) sulfossuccinato], Triton X-100 (etoxilato de octilfenol), Span 80 (monooleato de sorbitano) e Tween 80 (monooleato etoxilato de sorbitano) para a produção de biodiesel a partir de óleo de cozinha residual, utilizando metanol. O surfactante que apresentou melhor desempenho foi o Span 80, conduzindo a um teor de éster de 90,2\% para 96,3\% utilizando 0,03 g mL-1 de surfactante.

Em 2020, pesquisadores estudaram a utilização do surfactante 3-(N, Ndimetilmiristilamônio) propanossulfonato (3DMAPS, um surfactante zwitteriônico) na reação de transesterificação in situ de micro-organismos com o metanol. Neste trabalho, uma levedura oleaginosa, a biomassa úmida de Rhodotorula glutinis, foi utilizada diretamente na transesterificação in situ sem secagem da biomassa. Com a utilização do surfactante, houve um aumento do rendimento de FAME de 69\% para $83 \%$, em condições otimizadas de $50 \mathrm{mM}$ de surfactante, a uma razão molar de metanol:óleo de 10:1, com 10 h de reação a uma temperatura de $90^{\circ} \mathrm{C}$ (Chen et al. 2020).

Em estudo mais recente, pesquisadores utilizaram o surfactante Nonilfenol Sete Etoxilatos (NP7EO), na reação de transesterificação, obtendo um teor de éster de 98,95\%, com 5\% em peso de catalisador, $1 \%$ em peso de surfactante, com razão molar metanol:óleo de soja de 7:1, em 3 h de reação, a $65^{\circ} \mathrm{C}$ (Andrade et al., 2021).

A prospecção tecnológica é uma importante ferramenta para analisar o conhecimento em áreas específicas de forma clara e objetiva, fornecendo a situação atual das produções já realizadas em determinadas áreas. É o primeiro passo a ser realizado durante o desenvolvimento de projetos e pesquisas científicas, já que define as diretrizes gerais para a prática de uma pesquisa inovadora. Neste contexto, avaliando o cenário mundial, o presente artigo possui como objetivo prospectar tecnologias relacionadas com o uso de surfactantes em biodiesel, principalmente na área de surfactantes naturais, onde foi demonstrado a partir da busca, um número extremamente baixo de patentes sobre o tema. 


\section{Metodologia}

O presente projeto foi realizado em julho de 2021, sendo um estudo prospectivo, de análise quantitativa e exploratória através da busca de documentos de patentes que utilizaram surfactantes para a melhoria do processo de produção do biodiesel. O levantamento das patentes foi realizado a partir do banco de dados do European Patent Office (EPO), e do Instituto Nacional de Propriedade Industrial (INPI). Os pedidos de patentes encontrados foram depositados no período de 2000 a 2020. Os resultados das buscas que não apresentaram assuntos relacionados com a utilização de surfactantes no biodiesel foram descartados.

A prospecção iniciou com combinações das palavras-chave "biodiesel and surfactant* and natural", "biodiesel and surfactant* and additive*" e "biodiesel and surfactant*" no título e/ou resumo dos documentos. Com o objetivo de fazer uma busca mais específica, utilizou-se concatenações entre as palavras-chave com a inclusão do conector "and". Os resultados encontrados no banco de dados foram examinados, selecionados e em seguida exportados para o software Microsoft Office Excel, possibilitando realizar a plotagem dos gráficos e a análise da distribuição dos documentos de acordo com a Classificação Internacional de Patentes (CIP), a análise da evolução anual de depósitos de patentes e os países e empresas depositantes.

\section{Resultados e Discussão}

A prospecção tecnológica possui a finalidade de mapear as tecnologias existentes, que são capazes de influenciar a sociedade e economia. Na prospecção é possível identificar nas patentes, seus pesquisadores e empresas depositantes, países de origem, ano de publicação e suas classificações internacionais.

A partir da análise das palavras-chave "biodiesel and surfactant* and natural", "biodiesel and surfactant* and additive*" e "biodiesel and surfactant*", foi avaliado a quantidade de patentes depositadas tanto no banco de dado da EPO quanto no banco de dados do INPI, apresentado na Tabela 1.

Tabela 1. Número de patentes por palavras-chave recuperadas no banco de dado depositados no EPO e INPI, entre os anos de 2000 a 2020.

\begin{tabular}{|c|c|c|}
\hline Palavras-chave & EPO & INPI \\
\hline Biodiesel and surfactant $*$ and natural & 1 & 0 \\
\hline Biodiesel and surfactant $*$ and additive $*$ & 8 & 1 \\
\hline Biodiesel and surfactant $*$ & 90 & 5 \\
\hline
\end{tabular}

Fonte: Autores (2021).

A partir do estudo dos dados apresentados na Tabela 1, é possível verificar que a combinação de palavras-chave "biodiesel* and surfactant* and natural" e "biodiesel and surfactant* and additive*", possuem um número reduzido de patentes, tanto no banco de dado do EPO quanto no do INPI. Com isso, para poder fazer uma análise com um maior número de dados, foi necessário reduzir a especificidade, generalizando mais os termos, e usando apenas a combinação de palavras-chave "biodiesel and surfactant*", aumentando o campo de busca. Foram encontrados um total de 90 documentos no banco de dado do EPO e 5 documentos no banco de dados do INPI.

Após lido os resumos dos 5 documentos encontrados no banco de dados do INPI, com a combinação de palavras- 
chave "biodiesel and surfactant*", apenas 2 são pertinentes ao tema de estudo. Uma patente, depositada em 2011, pela indústria TerraVia Holdings, que estuda a utilização de célula microbiana oleaginosa como surfactante, e a outra patente, depositada em 2007, pela Universidade Federal do Rio de Janeiro, que pesquisa a utilização do carbonato de glicerina como surfactante.

Como o banco de dados do European Patent Office apresentou um número significativamente maior do que o do Instituto Nacional de Propriedade Industrial, na combinação de palavras-chave "biodiesel and surfactant*", para a análise e plotagem dos gráficos foram utilizadas as patentes depositadas no banco de dados do EPO. Após a realização da leitura dos resumos dos 90 documentos, apenas 50 foram selecionadas pertinentes ao tema do estudo.

Os documentos estudados estão distribuídos entre cinco seções da Classificação Internacional de Patentes (CIP): A necessidades humanas, B - operações de processamento; transporte, $\mathrm{C}$ - química; metalúrgica, E - construções fixas e F engenharia mecânica; iluminação; aquecimento; armas; explosão. A partir da Figura 1 é possível ressaltar que a maior utilização das patentes selecionadas, 76\%, estão relacionadas na seção C, ou seja, na área de química e metalúrgica.

Figura 1. Distribuição de patentes de acordo com a Classificação Internacional de Patentes (CIP).

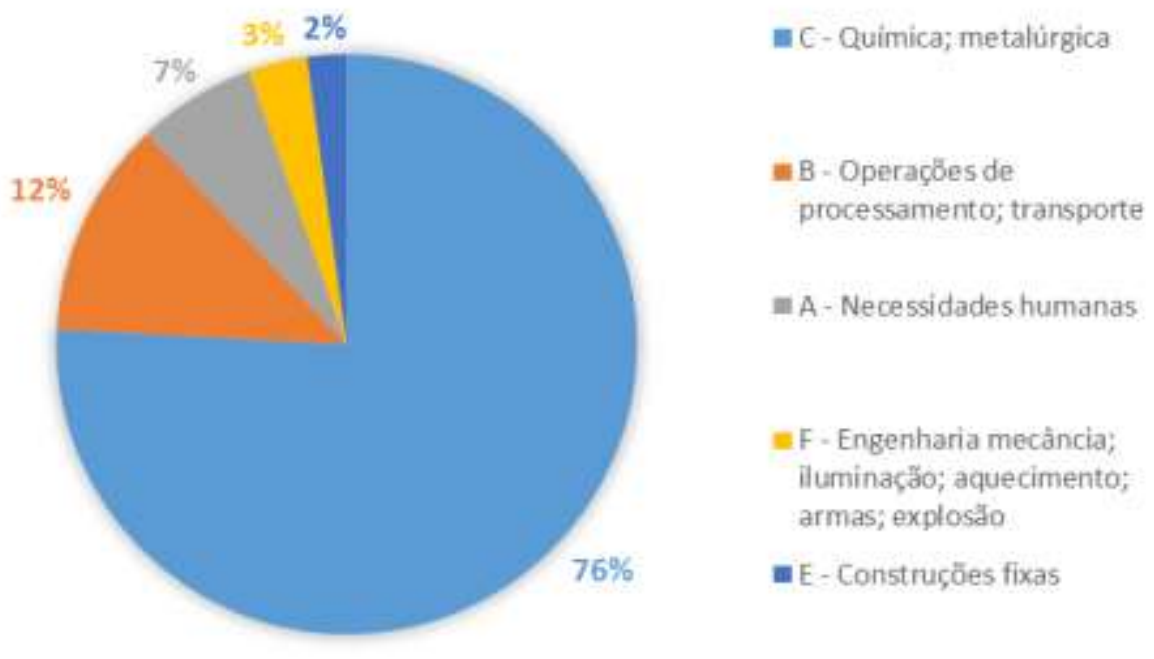

Fonte: Autores (2021).

No presente estudo, foram encontradas 91 CIP. A Figura 2 mostra os 12 códigos mais aplicados e a quantidade de seus respectivos depósitos. É possível verificar que todos os 12 códigos selecionados estão relacionados na área de química e metalúrgica, sendo que 85\% estão focados em combustíveis carbonáceos líquidos (C10L1) e 15\% estão relacionados com gorduras, óleos ou ácidos graxos combinados da modificação química de gorduras, óleos, ou ácidos graxos (C11C3). Dos documentos selecionados $22 \%$ estão direcionados com inovações voltadas para componentes que consistem em carbono, hidrogênio e oxigênio apenas (C10L1/02). O total de 42\% (soma de C10L1/04 + C10L1/10 + C10L1/18 + C10L1/182 + C10L1/185 + C10L1/19) dessas inovações estão relacionadas com adição de componentes ao biodiesel.

É importante ressaltar que uma patente pode receber mais de uma classificação, ou seja, ter mais de um código CIP, e quando não há um CIP que descreva exatamente o estudo, utiliza-se o código que mais se assemelhar (INPI, 2021). 
Figura 2. Número de patentes recuperados no banco de dados do EPO por Classificação Internacional de Patentes (CIP).

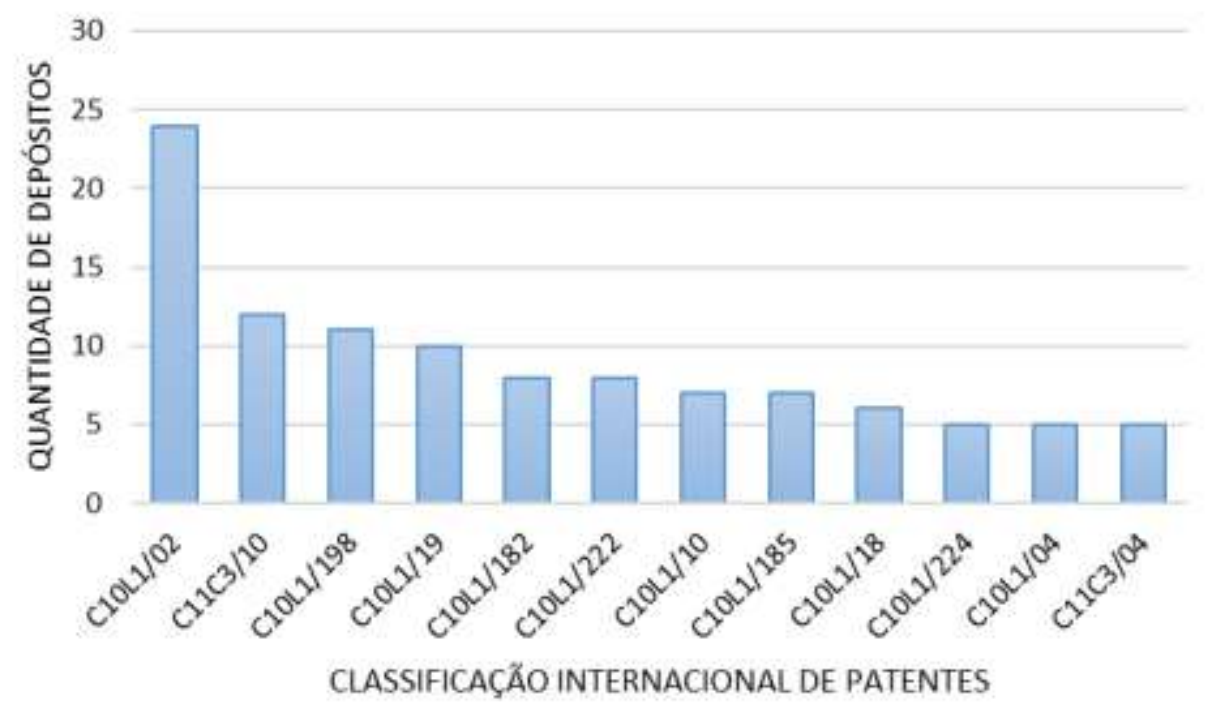

Fonte: Autores (2021).

A Figura 3 apresenta a quantidade anual de depósitos nos últimos 20 anos. Em 2005 houve o aumento da porcentagem do biodiesel utilizado no diesel a base de petróleo, na União Europeia foi de $2 \%$ por exemplo (Biodieselbr, 2012), podendo explicar o fato do aumento do número de patentes a partir de 2008. Um outro fato que pode explicar esse aumento a partir de 2008 foi a implementação de quatro novos programas chineses em 2007, que incentivam o estudo e implementação de novas matrizes energéticas mais limpas e obtidas de fontes renováveis, são eles o "Energy Conservation Law", "Nacional Assessment Report on Climate Change", "Renewable Energy Law" e o "China's Nacional Climate Change Program" (Rocha, Torre \& Quintella, 2013). Os anos que tiveram um maior número de documentos depositados foram 2014 e 2013, com 13 e 6 documentos respectivamente. Sendo que em 2013, a produção mundial do biodiesel teve um aumento de 13\% (Fernandes et al., 2015). Esse aumento também pode estar correlacionado com o fato da crescente busca de matrizes energéticas obtidas a partir de fontes renováveis e que possibilitem redução da emissão de gases poluentes. A queda da quantidade de patentes em 2015 pode estar associada com a desaceleração econômica de alguns países, principalmente a China, ocasionando volatilidade aos mercados globais. Inclusive, em 2015, em uma tentativa de estimular a economia, aumentando as exportações, o Banco Popular da China, chegou a desvalorizar o yuan (moeda chinesa) em relação ao dólar (Lima, 2015).

De acordo com a análise da Figura 3, é possível notar que não há hegemonia em relação a quantidade de patentes depositadas por ano. Além disso, é possível perceber que nos últimos anos houve uma queda do número de patentes, sendo este um fator intrigante e preocupante, já que a redução da quantidade de depósito de patentes pode indicar uma diminuição e estagnação no desenvolvimento tecnológico do biodiesel. Como há um período de sigilo dos documentos de 18 meses, a redução nesse período pode ser explicada por esse fator. 
Figura 3. Número de patentes recuperados no banco de dados do EPO por ano de publicação.

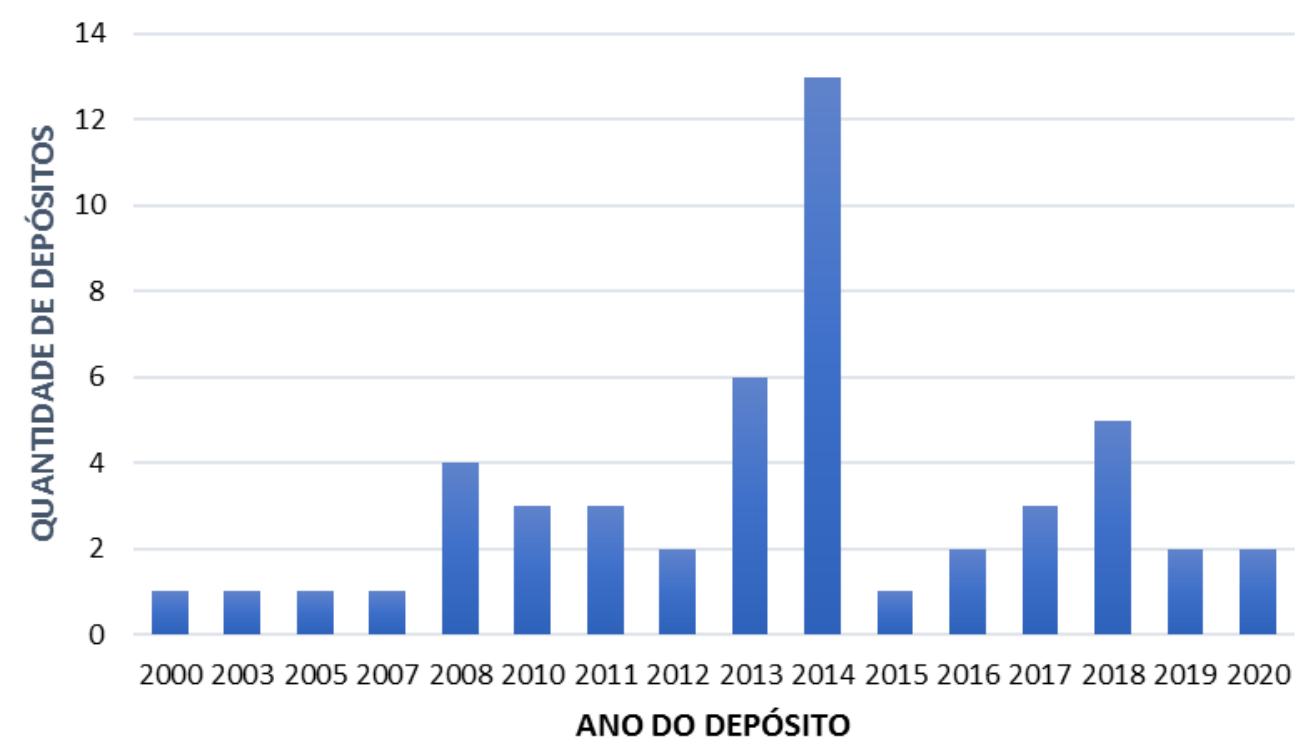

Fonte: Autores (2021).

A Figura 4 apresenta todos os países que depositaram as patentes do banco de dados da EPO. No total foram 7 países. O país que depositou significativamente o maior número de documentos foi a China (CN), com 40 patentes (80\%). Em seguida, foram os Estados Unidos da América (US), com apenas 3 depósitos (6\%); tanto a Organização Mundial da Propriedade Intelectual (WO) quanto o Japão (JP) depositaram 2 documentos cada, e tanto a República da Korea (KR), quanto o Canadá (CA) e a Ucrânia (UA), depositaram apenas um documento casa. É possível notar que graças ao seu constante aumento do desenvolvimento tecnológico sobre a utilização de surfactantes em biodiesel, a China é a maior detentora de patentes em comparação aos outros países. Na base de dados da EPO, não foi encontrada nenhuma patente depositada pelo Brasil relacionada com o tema, evidenciando falta de estudos e inovações sobre o uso de surfactantes no biodiesel, demonstrando ser uma área promissora para investimentos e desenvolvimento de novas tecnologias. 
Figura 4. Número de patentes recuperados no banco de dados do EPO por país de origem. Onde: UA - Ucrânia; CA - Canadá; KR - República da Coréia; JP - Japão; WO - Organização Mundial da Propriedade Intelectual; US - Estados Unidos da América; $\mathrm{CN}$ - China.

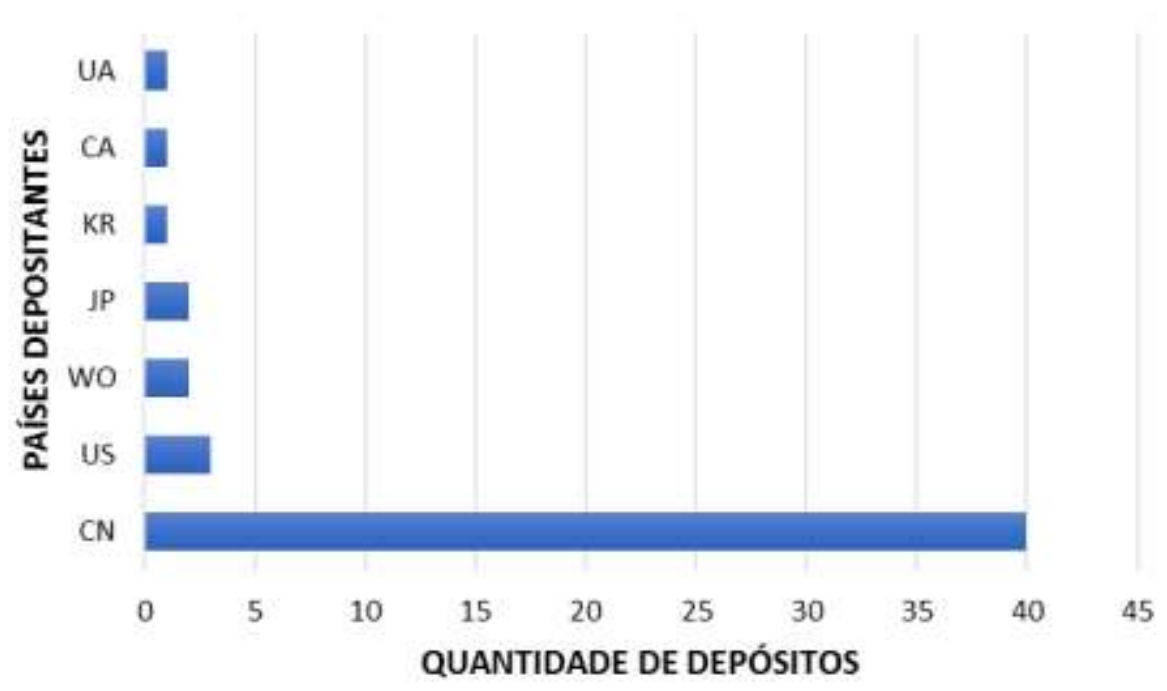

Fonte: Autores (2021).

Este trabalho, além de analisar as patentes em relação a suas classificações internacionais (CIP), ao país depositante e ano de publicação, analisou as patentes a partir dos setores da sociedade que são responsáveis pelo seu desenvolvimento e depósito no banco de dados do EPO (Figura 5). Os principais depositantes encontrados foram as indústrias, universidades e autônomos. É possível verificar que a indústria foi responsável pelo maior número de depósitos, com 30 documentos (60\%). Esse dado pode indicar que a produção de surfactante utilizado no biodiesel é uma área de maior interesse industrial, e consequentemente comercial. A indústria chinesa, Foshan Tiansheng Long Grease Chemical Co Ltd, é a que possui o maior número de documentos, 6, todos depositados no ano de 2014. As universidades foram o segundo maior setor responsável pelos depósitos, em um total de 12 documentos (24\%), ressaltando que das 12 universidades que depositaram as patentes, 10 $(83,33 \%)$ são provenientes de universidades chinesas. Os autores autônomos depositaram 8 documentos (16\%), sendo que 6 dos autores são chineses (75\%), um autor é canadense e um autor é americano. 
Figura 5. Porcentagem da quantidade de patentes recuperados no banco de dados do EPO por empresas depositantes.

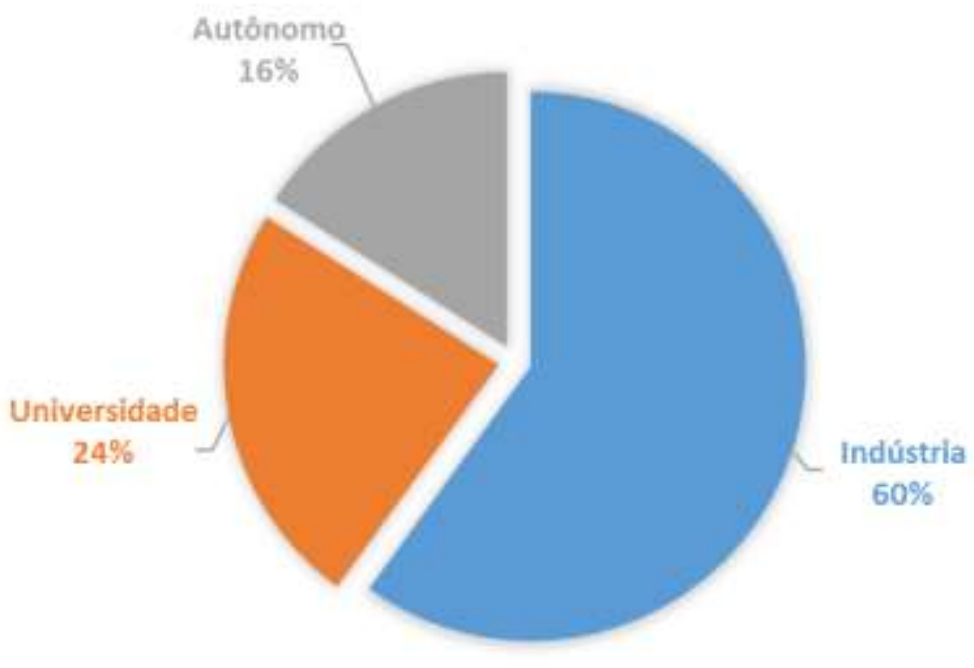

Fonte: Autores (2021).

Dos 50 documentos encontrados e selecionados no depósito da EPO, apenas um documento aborda sobre a utilização de surfactante natural na produção do biodiesel; uma patente produzida nos Estados Unidos, sobre a utilização do óleo vegetal como surfactante na produção do biodiesel, realizado pela indústria Oil Chem Tecnologies em 2013. Foi possível observar que existe uma necessidade de investir e realizar mais pesquisas e patentes sobre a produção e utilização de surfactantes naturais no biodiesel, já que a grande maioria de surfactantes sintéticos são tóxicos e cancerígenos. Os surfactantes naturais, além de serem mais seguros, são sustentáveis e rentáveis.

\section{Conclusão}

A partir da análise e realização da prospecção tecnológica, o presente trabalho demonstrou que há uma quantidade significativa de patentes depositadas no banco de dados da EPO, que estão relacionadas com o desenvolvimento de tecnologias de novos surfactantes, sintéticos e naturais, que podem ser utilizados na produção de biodiesel. Confirmando a existência de diversas pesquisas na área de aprimoramento de produção do biodiesel, já que este, por ser um combustível renovável e de menor emissão de gases de efeito estufa, é altamente promissor, acarretando em um maior interesse acadêmico, devido ao crescente aumento da demanda mundial para a utilização de combustíveis obtidos de fontes renováveis. Entretanto, apesar de existir uma boa quantidade de patentes, a grande maioria são provindas da China (80\%), não sendo encontrada nenhuma patente depositada no Brasil no banco de dados da EPO, demonstrando que esse tema é uma alternativa altamente promissora para investimentos brasileiros de pesquisa, desenvolvimento e apropriação tecnológica.

O presente trabalho indicou que o número de patentes de surfactantes naturais utilizados no biodiesel é extremamente pequeno no banco de dados do EPO (1 documento), e inexistente no banco de dados do INPI, provavelmente por ser uma área de pesquisa nova. Sendo necessário um maior investimento em pesquisas futuras concentradas no teste de substâncias naturais como aditivo na reação de transesterificação.

\section{Agradecimentos}

Os autores agradecem o apoio financeiro da Coordenação de Aperfeiçoamento de Pessoal de Nível Superior - Brasil (CAPES) - Código Financeiro 001. 


\section{Referências}

Andrade, M. R. A., Silva, C. B., Costa, T. K. C., Barros Neto, E. L. \& Lavoie, J. M. (2021). An experimental investigation on the effect of surfactant for the transesterification of soybean oil over eggshell-derived $\mathrm{CaO}$ catalysts. Energy Conversion and Management: $\mathrm{X}$, 11 , 100094 . https://doi.org/10.1016/j.ecmx.2021.100094.

Biodieselbr. (2021). Produção de biodiesel na união européia. https://www.biodieselbr.com/biodiesel/mundo/biodiesel-uniao-europeia.

Booma D. P., Joseph, D.R., Gokulnath, R., Manigandan, S., Gunasekar, P. \& Anand, T.P.P. (2020). The effect of TiO2 on engine emissions for gas turbine engine fueled with jatropha, butanol, soya and rapeseed oil. International Journal of Turbo \& Jet-Engines, 37(1), 85-94. https://doi.org/10.1515/tjj-2019-9018.

Brasil. (2021). Base de patentes. Instituto Nacional de Propriedade Industrial (INPI). http://www.inpi.gov.br

Chang, T., He, L., Zhang, X., Yuan, M., Qin, S. \& Zhao, J. (2015). Brönsted acid surfactant-combined dicationic ionic liquids as green catalysts for biodiesel synthesis from free fatty acids and alcohols. Chinese Journal of Catalysis, 36, 982-986. https://doi.org/10.1016/S1872-2067(15)60852-X.

Chen, S. J., Kuan, I. C., Tu, Y. F., Lee, S. L. \& Yu, C. Y. (2020). Surfactant-assisted in situ transesterification of wet Rhodotorula glutinis biomass. Journal of Bioscience and Bioengineering, 130 (4), 397-401. https://doi.org/10.1016/j.jbiosc.2020.05.009.

European Patent Office. (2021). Pesquisa de patentes. https://worldwide.espacenet.com/.

Fernandes, F. M., Silva, M. S., Lima, A. M. F., Rocha, A. M., Soares, P. M., Konish, F. (2015). Biodiesel no mundo e no brasil: situação atual e cenários futuros. Agrener gd, $10^{\circ}$ Congresso sobre Geração Distribuída e Energia no Meio Rural.

Fonseca, J.M., Teleken, J.G., Almeida, V.D.C. \& Silva, C. (2019). Biodiesel from waste frying oils: methods of production and puri fi cation. Energy Conversion and Management, 184, 205-18. https://doi.org/10.1016/j.enconman.2019.01.061.

Gomez, I. A. G., Gomes, K. B., Oliveira, V. I. M. X., Mattedi, S., Simonelli, G., Santos, L. C. L. \& Lobato, A. K. C. L. (2019). Effects of the addition of [2hea][hx] on biodiesel synthesis using methanol and methanol/ethanol mixture. Brazilian Journal of Petroleum and Gas, 13 (3), 175-186. http://dx.doi.org/10.5419/bjpg2019-0015.

Guimarães, A. K. V., Jesus, A. A., Oliveira, H. N. M., Barros Neto, E. L., Gondim, A. D \& Chiavone-Filho, O. (2018). Biodiesel from oiticica oil (Licaniarígida, Benth): production, thermogravimetric, and oxidative stability studies. Brazilian Journal of Petroleum and Gas, 12, 107-122. https://doi.org/10.5419/bjpg2018-0011.

INPI, Instituto Nacional da Propriedade Industrial. (2021). Manual Básico para Proteção por Patentes de Invenções, Modelos de Utilidade e Certificados de Adição. https://www.gov.br/inpi/pt-br/servicos/patentes/guia-basico/ManualbsicodePatentes20210607b.pdf.

Jagtap, S.P., Pawar, A.N. \& Lahane, S. (2020). Improving the usability of biodiesel blend in low heat rejection diesel engine through combustion, performance and emission analysis. Renewable Energy, 155, 628-44. https://doi.org/10.1016/j.renene.2020.03.115.

Kalavathy, G. \& Baskar, G. (2019). Synergism of clay with zinc oxide as nanocatalyst for production of biodiesel from marine Ulva lactuca. Bioresource Technology, 281 (434), 234-8. https://doi.org/10.1016/j.biortech.2019.02.101.

Leng, L., Chen, J., Leng, S., Li, W., Huang, H., Li, H., Yuan, X., Li, J. \& Zhou, W. (2019). Surfactant assisted upgrading fuel properties of waste cooking oil biodiesel. Journal of Cleaner Production, 210, 1376 - 1384. https://doi.org/10.1016/j.jclepro.2018.11.027.

Leng, L., Li, H., Yuan, X., Zhou, W. \& Huang, H. (2018). Bio-oil upgrading by emulsification/microemulsification: A review. Energy, 161,214 - 232. https://doi.org/10.1016/j.energy.2018.07.117.

Lima, D. (2015). Mesmo com o crescimento fraco, economia global surpreende analistas em 2015. https://agenciabrasil.ebc.com.br/economia/noticia/201512/mesmo-com-crescimento-fraco-economia-global-surpreende-economistas-em-2015.

Manigandan. S., Atabani, A.E., Kumar, P.V. \& Gunasekar, P. (2020). Impact of additives in Jet-A fuel blends on combustion, emission and exergetic analysis using a microgas turbine engine. Fuel, 276, 118104. https://doi.org/10.1016/j.fuel.2020.118104.

Mahlia, T.M.I., Syazmi, Z., Mofijur, M., Pgabas, A.E., Bilad, M.R., Ong, H.C. \& Silitonga, A.S. (2020). Patent landscape review on biodiesel production: Technology updates. Renewable and Sustainable Energy Reviews, 118, 109526. https://doi.org/10.1016/j.rser.2019.109526.

Mirhashemi, F.S. \& Sadrnia, H. (2020). NOX emissions of compression ignition engines fueled with various biodiesel blends: a review. Journal of the Energy Institute, 93 (1),129-51. https://doi.org/10.1016/j.joei.2019.04.003.

Narowska, B., Kułażyński, M., Łukaszewicz, M. \& Burchacka, E. (2019). Use of activated carbons as catalyst supports for biodiesel production. Renewable and Sustainable Energy Reviews, 135, 176-185. https://doi.org/10.1016/j.renene.2018.11.006.

Ramkumar, S. \& Kirubakaran, V. (2017). Biodiesel from vegetable oil as alternate fuel for C.I engine and feasibility study of thermal cracking: A critical review. Energy Conversion and Management, 118, 155-169. https://doi.org/10.1016/j.enconman.2016.03.071.

Rocha, A. M., Torre, E. A. \& Quintella, C. M. 2013. Biodiesel na república popular da china: mapeamento de artigos e patentes. Cadernos de Prospeç̧ão, 6, 302-311. https://doi.org/10.9771/cp.v6i3.11411.

Rodriguez, G. \& Beckman, E. J. (2020). Modelling phase behavior of triacylglycerol, diacylglycerol and monoacylglycerol related to biodiesel transesterification in mixtures of alcohols and $\mathrm{CO} 2$ using a polar version of PC-SAFT. Fluid Phase Equilibria, 503, 112303. https://doi.org/10.1016/j.fluid.2019.112303. 
Research, Society and Development, v. 10, n. 11, e389101119742, 2021

(CC BY 4.0) | ISSN 2525-3409 | DOI: http://dx.doi.org/10.33448/rsd-v10i11.19742

Salam, K. A., Velasquez-orta, S. B. \& Harvey, A. P. (2016). Surfactant-assisted direct biodiesel production from wet Nannochloropsis occulata by in situ transesterification/reactive extraction. Biofuel Research Journal, 9, 366-371. https://doi.org/10.18331/BRJ2016.3.1.6.

Simonelli, G., Moraes, C., Pires, C. A. de M. \& Santos, L. C. L. (2019). Multivariate study and optimization of biodiesel production using commercial surfactants. Chemical Industry and Chemical Engineering Quarterly, 25 (2), 183-192. https://doi.org/0.2298/CICEQ180318031S.

Trindade, M.E.J., Mattedi, S., Simonelli, G. \& Santos L.C.L. (2019). Use of protic ionic liquid ([deta][hx]) as co-solvent in biodiesel production. Brazilian Journal of petroleum and Gas, 13 (1), 057-065. http://dx.doi.org/10.5419/bjpg2019-0006.

Valverdea, A., Osmierib, L. \& Recasens, F. (2019). Binary interaction parameters from reacting mixture data. Supercritical biodiesel process with CO2 as cosolvent. The Journal of Supercritical Fluids, 143, 107-119. https://doi.org/10.1016/j.supflu.2018.05.023.

Wong, K.Y., Ng, J.H., Chong, C.T., Lam, S.S. \& Chong, W.T. (2019). Biodiesel process intensification through catalytic enhancement and emerging reactor designs: A critical review. Renewable and Sustainable Energy Reviews, 116, 109399. https://doi.org/10.1016/j.rser.2019.109399. 